\title{
Technical Study on 5G Using Soft Computing Methods
}

\author{
J. Divakaran, ${ }^{1}$ Somashekhar Malipatil, ${ }^{2}$ Tareeq Zaid, ${ }^{3}$ M. Pushpalatha, ${ }^{4}$ Vilaskumar Patil, ${ }^{5}$ \\ C. Arvind, ${ }^{6}$ T. Joby Titus, ${ }^{7}$ K. Srihari ${ }^{10},{ }^{8}$ M. Ragul Vignesh, ${ }^{9}$ Baswaraj Gadgay, ${ }^{10}$ \\ and Venkatesa Prabhu Sundramurthy $\mathbb{I D}^{11}$
}

\author{
${ }^{1}$ Department of Electronics and Communication Engineering, K.S.R. Institute for Engineering and Technology, Tiruchengode, \\ Tamil Nadu, India \\ ${ }^{2}$ Department of Electronics and Communication Engineering, Malla Reddy Engineering College and Management Sciences, \\ Medchal, Telangana 501401, India \\ ${ }^{3}$ Department of Electronics and Communication Engineering, Vardhaman College of Engineering, Hyderabad, \\ Telangana 501218, India \\ ${ }^{4}$ Department of Electronics and Communication Engineering, Siddartha Institute of Engineering and Technology, \\ Ibrahimpatnam, Rangareddy 501506, India \\ ${ }^{5}$ Department of Electronics and Communication Engineering, Faculty of Engineering and Technology (Co-Education), \\ Sharnbasva University, Kalaburagi, Karnataka 585103, India \\ ${ }^{6}$ Department of Electronics and Communication Engineering, Karpagam College of Engineering, Coimbatore, India \\ ${ }^{7}$ Department of Electronics and Communication Engineering, Dhanalakshmi Srinivasan College of Engineering, \\ Coimbatore, India \\ ${ }^{8}$ Department of Computer Science Engineering, SNS College of Technology, Coimbatore, India \\ ${ }^{9}$ Department of Computer Science Engineering, Dhanalakshmi Srinivasan College of Engineering, Coimbatore, India \\ ${ }^{10}$ Department of PG Studies, Visvesvaraya Technological University (VTU), Regional Campus, Kalaburagi, India \\ ${ }^{11}$ Department of Chemical Engineering, Addis Ababa Science and Technology University, Addis Ababa, Ethiopia
}

Correspondence should be addressed to Venkatesa Prabhu Sundramurthy; venkatesa.prabhu@aastu.edu.et

Received 25 October 2021; Revised 12 November 2021; Accepted 30 November 2021; Published 7 January 2022

Academic Editor: M Pallikonda Rajasekaran

Copyright $\odot 2022$ J. Divakaran et al. This is an open access article distributed under the Creative Commons Attribution License, which permits unrestricted use, distribution, and reproduction in any medium, provided the original work is properly cited.

With increasing advancements in the field of telecommunication, the attainment of a higher data transfer rate is essentially a greater need to meet high-performance communication. The exploitation of the fuzzy system in the wireless telecommunication systems, especially in Fifth Generation Mobile Networks (or) 5G networks is a vital paradigm in telecommunication markets. A comprehensive survey is dealt in the paper, where it initially reviews the basic understanding of fuzzy systems over 5G telecommunication. The literature studies are collected from various repositories that include reference materials, Internet, and other books. The collection of articles is based on empirical or evidence-based from various peer-reviewed journals, conference proceedings, dissertations, and theses. Most of the existing soft computing models are streamlined to certain applications of 5G networking. Firstly, it is hence essential to provide the readers to find research gaps and new innovative models on wide varied applications of $5 \mathrm{G}$. Secondly, it deals with the scenarios in which the fuzzy systems are developed under the 5G platform. Thirdly, it discusses the applicability of fuzzy logic systems on various $5 \mathrm{G}$ telecommunication applications. Finally, the paper derives the conclusions associated with various studies on the fuzzy systems that have been utilized for the improvement of $5 \mathrm{G}$ telecommunication systems. 


\section{Introduction}

5G telecommunication offers a role on high level flexibility and a high level scalable architecture that allows to connect all and everywhere. It offers additional support for network with a core network. It allows the development of new value by supporting new services based on 3 key usage domains: massive machine type communication (mMTC), enhanced mobile broadband (eMBB), and reduction of noise signals [1-5]. Hard QoS assurances such as bandwidth limits, packet loss and durability, and a nanosecond-level synchronization [1] will be accomplished. The support is focused mainly on integration in the standards set defined by the TSN task group IEEE 802.1 [6] for the IEEE Time Sensitive Network (TSN). TSC is a networking facility that offers high efficiency and availability for decision-making and/or isochronous communication. Initial deployments of narrowband radio (NR) have been undertaken at the late 2019, with the focus on the use of the 3GPP version of Release 15 (Rel-15). For mMTC, NR is accomplished by Narrow Band Internet of Things (NBIoT) machine-type communications technologies in 3GPP that provide incomparable low-power broad-ranging performances that cover the wide spectrum of data rates and implementation scenarios [7-11].

(i) Proximity Services: proximity support services (ProSe) are useful for both public security and business services. The key goal of the work of the Rel17 in this field [14] is to establish a standard public security and ProSe commercial architecture. For public protection, it is crucial to help ProSe exploration and coordination where the EU is not covered, e.g., in rural regions lacking network access in cases of disaster relief.

(ii) Multimedia Broadcast Services (MBMS): MBMS Support for NR will be implemented primarily in cases including the use of public security [15]. These cases involve transmission/multidiffusion over a region that may be broader than a single cell. An enabling feature will be a content delivery mechanism and content distribution infrastructure for multibase stations in an MC-PTM area. In the radios, it ensures wide deployment of user equipment (UE); hence, autonomous broadcasting is required to take place in each cell, rather than having specific support for the service of a broad-based single frequency network (SFN).

\section{Fuzzy Logic: Outline}

The Fuzzy Logic (FL) method is an input data vector nonlinear mapping into a scaled output that allows numerical and linguistic information to be processed and handled simultaneously. The FL will address true, false, or intermediate truth value statements. These assertions cannot be quantified with conventional mathematics. The configuration of the Fuzzy Logic Controller (FLC) consists of four elements, namely, fuzzification, knowledge base, inference mechanism, and defuzzification interface: (i) For mixing crisp values with language variable rules that have their corresponding fuzzy sets, the fuzzifier is essential.

(ii) The rules can be made available by an expert or derived from numerical evidence. As a set of IFTHEN sentences, the rules are represented in engineering cases.

(iii) The fuzzy product is inferred by the inference engine based on fused input and fused rules.

(iv) Crisp numbers are given on the defuzzifier map outputs.

\section{5G Fuzzy Telecommunication Systems}

In this section, we discuss the entire fuzzy application on $5 \mathrm{G}$ telecommunication systems over various operations that include handover management, traffic management, resource allocation, optimization models, and its relation with other technologies.

3.1. Handover Operation. A 5G heterogeneous network neuro-fuzzy controller was used to enhance the transfer operation. The controller architecture and variable input and output were developed. The controller has considered a rule base and mathematical templates. By conducting the computer simulation [14], the performance of the neurofuzzy transmission controller was tested.

For mobility control on small-cell networks, a complex fuzzy Q-learning algorithm is used. Initially, there were no fluorescent laws. In order to strike the equilibrium between signaling costs caused by transmission and user experience influenced by call decreases, the algorithm creates new fuzzy rules and obtains the necessary parameters through machine education. The performance is measured in an LTE simulator, and the effect of the EU speed is taken into account. The simulation findings demonstrate the utility of the algorithm to minimize the number of transmissions while keeping a minimal call drop ratio [15].

In [12], the optimum weight selection algorithm for network features, facilities, and consumer preferences is derived. These weights are used for an optimal applicant list in an intuitive trapezoidal fuzzy handover system.

A SDN-based transfer architecture is built in [13] that allows users and applications, including physics, to perceive global network statements and specifications from all viewpoints. In order to provide differentiated services, a context-aware multicriteria transfer process is built into the SDN controller. In view of the many complicated variables, a decision to transfer is taken based on a hierarchical fluctuated method of reference to process diverse natural-language qualities and fluent knowledge.

In [13], we created a software-defined networking (SDN) architecture, which allows users and applications to view global network statements and needs from all angles, including the physical layer. Then, the SDN controller is designed to provide differentiated services through a context-conscious multicriteria delivery system. A hierarchical fuzzy-inference scheme is used to manipulate diverse 
attributes and fuzzy natural-language knowledge, taking into account the many complicated variables.

In [16], an empirical model is established for determining the time spent in the vehicle in a beam coverage. If all small mmWave cells are available with multiple connections during a transfer case, then we deduct the longest stay time from all possible connections that represent the theoretical limit of the stay time efficiency. We then developed a beamcentered decision algorithm based on fuzzy logic to maximize the time spent in the car. Simulation tests are carried out in order to verify our analytical model and to demonstrate our FL-based solution performance relative to the most widely used relation method.

A protocol is built in [17] to mitigate the vulnerabilities which make it susceptible to jamming, DoS attacks, missing key confirmatory attacks, traceability attacks, and replay attacks. Any such attacks were prevented by the developing specification; the use of that protocol is clear since it maintains the traditional $5 \mathrm{G}$ architecture. In terms of performance, this protocol has comparatively small size complexity and delays in handling.

3.2. Optimization Models. In [18], the approach for the software-based network controller is defined to resolve multitarget resource optimization problems for $5 \mathrm{G}$-powered VANETs by a hybrid fuzzy logic-directed genetic algorithm. The suggested solution formulates five separate network capital optimization scenarios in 5G VANETs, in order to realize a service-oriented view. In addition, according to the type of service needs of users, a suggested fuzzy inference scheme optimizes the weights of the multitargets. The solution suggested shows the minimum value in comparison with the GA for the multiobjective cost function. Compared with other systems, the simulation findings indicate the minimum importance of end-to-end delays. The solution would allow network service providers to incorporate a customized network architecture in accordance with the diverse customer needs. The methods required to optimize the models are shown in Figure 1.

In [19], a method for optimizing transfer control parameters (HPs) using weighed fuzzy self-optimization (WFSO) was developed. The determination of the $\mathrm{HO}$ in this context depends on the three attributes: the signal-to-interference-plus-noise ratio, the service load, and the base station target traffic, as well as the speed of user equipment. The self-optimized HCPs (the $\mathrm{HO}$ margin and time to trigger) have been updated to increase the $\mathrm{HO}$ value based on the current state of the attributes. The simulation results show that, when compared to other literature algorithms, the WFSO solution significantly reduces the rates of HOPP, radio connection loss, and $\mathrm{HOF}$.

The study of $5 \mathrm{G}$ infrastructure for a Microgrid Shipboard wireless network control system is studied in [20]. In this analysis, oscillation damping is created using $2^{\text {nd }}$ order fractional fuzzy with an adaptive neuro-fuzzy inference system (ANFIS) method. The interface parameters are set at an optimal rate using the Sine Cos algorithm (SCA).
In [21], a furious logic-based game-theoretical paradigm is being used to resolve these problems and to analyze changes in 'HetNets' energy production. For transfer decisions, we design floated inference rules and the selection of target base stations by means of an efficient ranking methodology that takes into account both energy/spectral performance and overhead signaling at the same time.

An energy-efficiency enhancement logic-based gametheoretical paradigm in HetNets is analyzed [22]. In addition to consideration of energy/spectral quality and handover performance, the analysis established flawless judgment rules for transitions and target base station placement. In particular, for the limited number of active users and fast usage speeds, our findings show that energy usage has significantly increased as well as the management of handovers and cell loads.

3.3. Fuzzy Resource Allocation. A user-driven contextaware architecture is developed in [23] for the selection of networks in multiradio access (MRA) technologies. It depends on fuzzy reasoning to deal with the loss of knowledge normally linked to the end point and the radio environment inherent randomness. In particular, the first step of a fuzzy logic controller is to estimate that each RAT is out of context suitable to meet the QoS needs of a number of heterogeneous applications. In order to merge these calculations with different context components (e.g., terminal capacities, user needs, and operator policies), the fuzzy multiattribute decision-taking (MADM) technique is introduced in order to infer the in-context adequacy of each RAT. Based on this new metric, the best RAT in a given setting can be selected using two spectrum selection and spectrum mobility functions. The fuzzy MADM method is validated to carry out a context-aware download for a mixture of timesensitive and efficient applications in a dense small-cell setting.

The mmWave network in [24] has a jumbled logically dependent relay selection system. The Flu-cyclical logic system is used for relay selection systems that select the best relay mechanism among the access point neighbors based on the three parameters that include data rate, node speed, and battery level when the mmWave link is blocked between the $\mathrm{AP}$ and the mobile node.

A mechanism for spectrum sharing is focused on furious software to enable MNOs to choose the most appropriate means of resource sharing to maximize the distribution of resources [25]. A new collaboration mechanism allows MNOs and other spectrum licensees to share information on spectrum infrastructure and to share several options for the potential solution.

3.4. Traffic Management. In [26], the user equipment services in the $5 \mathrm{G}$ wireless network are allocated by the fuzzy control algorithm. Multimedia data are nowadays the preferred traffic on the Internet and have helped to increase the amount of data. In this investigation, 


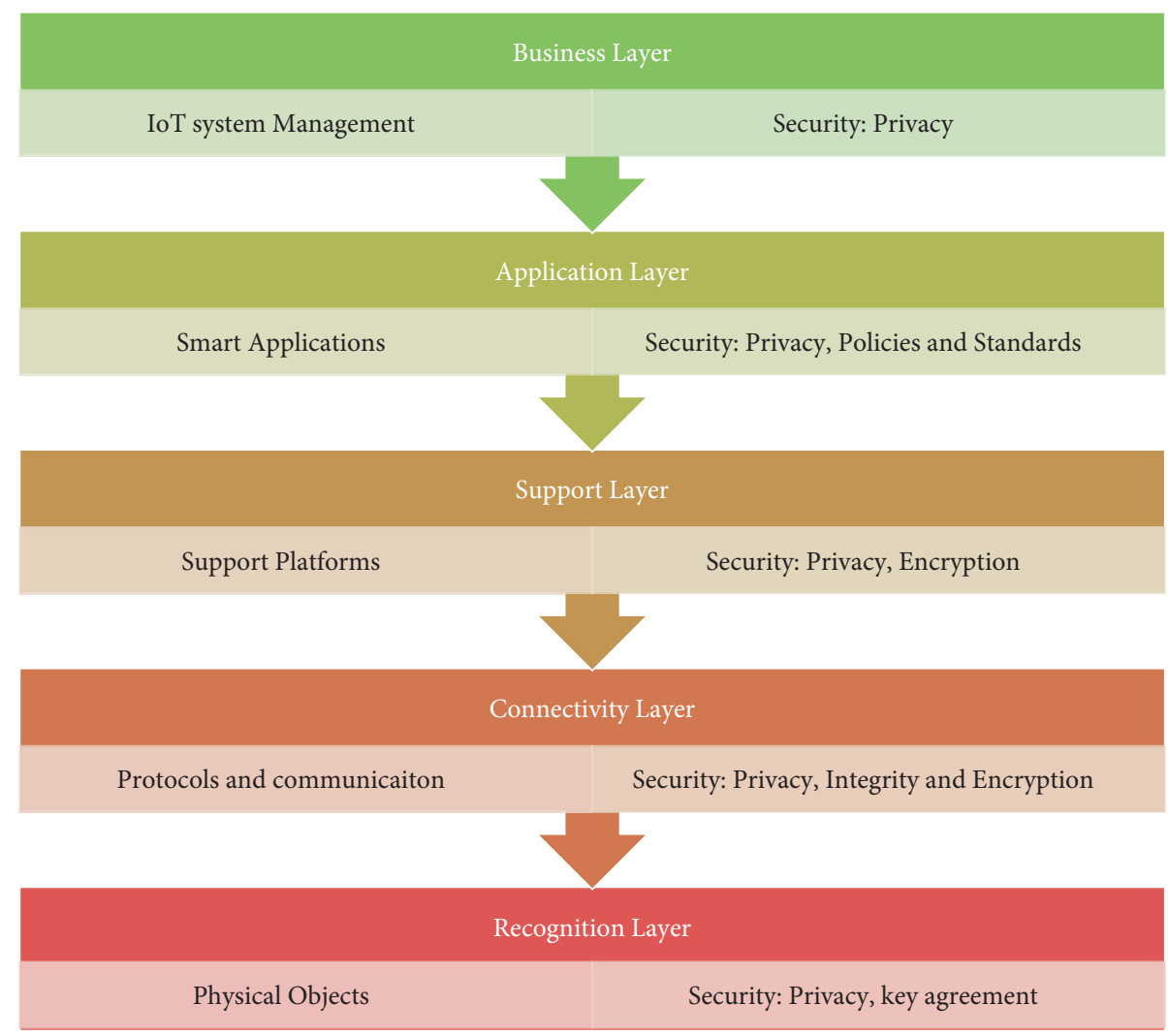

FIgURE 1: Optimistic approaches in various domains of 5G.

however, forms of inquiries are examined in real time (RT) and nonreal time (NRT). A cellular base station gNodeB (gNB) of $5 \mathrm{G}$ networks is used for the Radio Resource Control (RRC) connectivity and collaboration protocol. RRC operations are managed by the fuzzy algorithm management. For various configurations of inputs and outputs, fuzzy rules are planned, implemented, and evaluated. The first queue planning of RT and NRT requests is used for processing. It is compared with the programming for First Come First Service (FCFS). The average reaction time is 40 percent less than FCFS for RT First queue scheduling.

A network selection system is to adjust the relevance of each provider to the patient's health status [27]. The scheme consists of two FMADM algorithms: the Trapezoidal Fuzzy Adaptive Analytical Network Process (TF-AANP) estimates the speed of vehicle movement and rates it based on Trapezoidal Fuzzy TOPSIS with Adaptive Criteria Weights (TFT-ACWs) for achieving the rating of the applicant network. The scheme is based on two different FMADM algorithms. Both algorithms use trapezoidal fuzzy numbers with an interval value (IVTFNs).

The Enriched Neuro-Fuzzy (ENF) classifier is suggested to be used in traffic analysis in [28]. The primary load balancing machine and the secondary load balancing machine are used. This aircraft balances the load between the controllers. Switch conversion is presented for controller load balancing. The entropy feature predicts the overloaded controller. The Fitness-Based Reinforcement
Learning (F-RL) algorithm then makes a decision about migration.

3.5. Other Technologies. The method in [29] examined the fuzzy model of a power buffer prediction control synthesis, which is operated by a network of low latency contact such as that imagined in the 5th generation. A DC microgrid is dynamic stable. The solution developed is based on a TakagiSugeno fuzzy model, a floating observer, and a model predictive system to mitigate the impact on DC MG plant reaction of the $5 \mathrm{G}$ network. The solution is resilient to network delays because of the use of two NDCs and results in little computational pressure.

The cluster, aggregate, and classification framework of [30] means that the mobile network radio resource is used more efficiently, that the signal charge to the base station is reduced, and that M2M devices are powered. The new feature of the algorithm is that it selects the principal mobile node based on the Voronoi chart and on the fuzzy logic methods. In order to move data into $4 \mathrm{G} / 5 \mathrm{G}$ base stations, the latest algorithms are introduced on M2M gates that carry out the main node selection functions, divide different data into service groups, and balance loads from gates.

The Network Selection Algorithm was introduced by Skondras et al. [31] and is called the Dynamic Trapezoidal Fuzzy TOPSIS. In accordance with the seriousness of a natural catastrophe, the corresponding selection criteria into 
the ranking model candidate networks take each operation. The criterion assessment is based on interval-valued trapezoidal fuzzy numbers.

\section{Limitations}

As a result of this, most soft computing strategies concentrate on training data that include ambiguous or incomplete information. In a sense, the human mind serves as a model for soft computing. There are three principles that guide software development: utilize tolerance for ambiguity, uncertainty, and partial truth for tractability and low-cost solution. When used in conjunction with other soft computing approaches such as visualization, this technique can be quite useful because of the complementary nature of them. The two can work together to solve issues that traditional mathematical methods cannot handle because they are either too intricate or noisy.

The design of self-healing and intelligent networks has been examined from many angles; however, there are still a number of outstanding issues: how can you get to a network that is completely self-sufficient? How might the trade-offs between efficiency and cost/complexity be best explored?

\section{Future Work}

The demand on 5G telecommunication networks in providing access to new applications and mass connectivity can be presented in terms of directions for future scope. Hence, the challenges related to the driving technologies and support systems or hardware should enable a higher level of QoS.

The 5G high-speed network architecture enables information transfer and network communication. A new architecture for big data needs to get developed to leverage the future gain from big data in 5G IoT. This architecture can handle a vast volume of data and incorporate the big data chain into the network through data collection, storage, retrieval, and analysis to improve the network operation. Under this new system, unused data should be ignored, and the desired services should get processed at the appropriate position. The other part of big data science is personalized big data processing networking. With this Service Function Chain (SFC) solution or network slicing, many large data networks may be supported by providing service-oriented networking across a physical network infrastructure. A part of the end-to-end network may also be customized to meet the service specifications. Service Feature Chain (SFC) should get tuned to allow the optimal use of networking tools. The SFC needs to be in a position to respond and assess improvements in network and infrastructure conditions in favor of 5G.

The collection of waveforms is one of the most difficult tasks for developing modern $5 \mathrm{G}$ radio (NR). Due to its high interchannel (ICI), high intersymbol interference (ISI), and high PAPR, OFDM was the first choice in developing LTE, but it cannot be appropriate for the 5-G waveform. The research obstacles for $5 \mathrm{G}$ waveforms are the constraints of OFDM-based waveforms. A shorter latency of less than $1 \mathrm{~m}$ is the first feature of the modern waveform for new services and applications. Low latency in IoT is used, and high latency is used in eMBB and critical connectivity, such as automotive autonomy and Internet of vehicles. The current waveform is also designed to operate a cyclical prefix. In two standard and expanded modes, the cyclic prefix can be used. The cyclic prefix alternative provides a short-lived structure. The selection of numerology is considered in $5 \mathrm{G}$ waveform architecture and uses various numerical values.

Due to an intensive study of energy use, the $5 \mathrm{G}$ wireless communication network has been a central pillar. Millions of devices will connect to more base stations than existing LTE networks in a single network architecture with 5G. Thus, the energy-efficient architecture and operation of the system are vital needs to accommodate this huge unit. The only way to solve energy efficiency is to use small-cell base stations. In the large-density consumer zones, the small base station aims to maximize power. The coverage is also improved; the data rate is increased, and the battery capacity is increased by lower power usage.

A heterogeneous network comes into being on the $5 \mathrm{G}$ broadband network. Catching and computing capital should be intelligent in favor of big data in heterogeneous networks in the form of $5 \mathrm{G} \mathrm{IoT}$. Competition in connectivity, capture, and computational capital is also important. To cut the coordination link, all these properties are used. The final calculation results should be temporarily stored, reducing the cost of storage. For optimal resource supply, the compromise in the 5G network between the 'HetNets' resources is thus essential. Because $5 \mathrm{G}$ IoT develops with a lot of data, these data are obtained from various resources, which result in an uneven distribution of data load. The solution for storing, recovering, and manipulating such a large volume of information is then cooperative edge capturing. The edge data processing capabilities are available.

To reduce the cost and physical size of the base station, a multiband power amplifier is needed for 5G IoT. This allows all wireless functions to operate concurrently, allowing multiband power amps to accommodate multiband frequency signals at the same time. The powerful power amplifier in the base station is an important element in mobile device growth.

\section{Conclusions}

This study presents a survey on various reviews that present the understanding of fuzzy systems over 5G. It further deals with the scenarios in which the fuzzy systems are developed under the $5 \mathrm{G}$ platform. It discusses the applicability of fuzzy logic systems on various $5 \mathrm{G}$ telecommunication applications. Furthermore, the study derives the conclusions associated with various studies on the fuzzy systems that have been utilized for the improvement of $5 \mathrm{G}$ telecommunication systems. We have shown how machine learning techniques can be used to design smarter future networks. Researchers looking at the usage of ML in future $5 \mathrm{G}$ networks will find our references to be particularly helpful in this regard. In addition, we have finished by pointing out the computational conditions for ML apps. In addition, the study raised a slew of questions for future networks that need answering. 


\section{Data Availability}

The datasets used and/or analyzed during the current study are available from the corresponding author on reasonable request.

\section{Conflicts of Interest}

The authors declare that there are no conflicts of interest.

\section{References}

[1] C. Ge, N. Wang, I. Selinis et al., "QoE-assured live streaming via satellite backhaul in $5 \mathrm{G}$ networks," in IEEE Transactions on Broadcasting, vol. 65, no. 2, pp. 381-391, 2019.

[2] A. Ijaz, L. Zhang, M. Grau et al., "Enabling massive IoT in 5G and beyond systems: PHY radio frame design considerations," in IEEE Access, vol. 4, pp. 3322-3339, 2016.

[3] A. Ghosh, A. Maeder, M. Baker, and D. Chandramouli, " $5 \mathrm{G}$ evolution: a view on $5 \mathrm{G}$ cellular technology beyond 3GPP release 15," in IEEE Access, vol. 7, Article ID 127639, 2019.

[4] J. Nightingale, P. G. Salva, J. M. A. Calero, and Q. Wang, "5GQoE: QoE modelling for ultra-HD video streaming in $5 \mathrm{G}$ networks," in IEEE Transactions on Broadcasting, vol. 64, no. 2, pp. 621-634, 2018.

[5] D. Sattar and A. Matrawy, "Optimal slice allocation in 5G core networks," in IEEE Networking Letters, vol. 1, no. 2, pp. 48-51, 2019.

[6] S. H. HosseiniNazhad, M. Shafieezadeh, and A. Ghanbari, "Efficient Non-orthogonal Multiple Access with Simultaneous User Association and Resource Allocation. Bulletin of the Polish Academy of Sciences," Technical Sciences, vol. 63, no. 3, pp. 665-675, 2019.

[7] P. Panagoulias, I. Moscholios, P. Sarigiannidis, M. Piechowiak, and M. Logothetis, "Performance metrics in OFDM wireless networks supporting quasi-random traffic," Bulletin of the Polish Academy of Sciences, Technical Sciences, vol. 68, no. 2, 2020.

[8] R. Gessing, "Whether the opinion about superiority of fuzzy controllers is justified," Bulletin of the Polish Academy of Sciences, Technical Sciences, vol. 58, no. 1, pp. 59-65, 2010.

[9] M. B. Gorzałczany and F. Rudziński, "Handling fuzzy systems' accuracy-interpretability trade-off by means of multi-objective evolutionary optimization methods-selected problems," Bulletin of the Polish Academy of Sciences, Technical Sciences, vol. 63, no. 3, pp. 791-798, 2015.

[10] P. Serkies, "A novel predictive fuzzy adaptive controller for a two-mass drive system," Bulletin of the Polish Academy of Sciences, Technical Sciences, vol. 66, no. 1, 2018.

[11] C. V. Nahum, L. D. N. M. Pinto, V. B. Tavares et al., "Testbed for $5 \mathrm{G}$ connected artificial intelligence on virtualized networks," in IEEE Access, vol. 8, Article ID 223202, 2020.

[12] M. Liu, Y. Huan, Q. Zhang, W. Lu, W. Li, and T. A. Gulliver, "Multiple attribute handover in 5G HetNets based on an intuitionistic trapezoidal fuzzy algorithm," in Proceedings of the 2018 IEEE/CIC International Conference on Communications in China, pp. 261-266, (ICCC Workshops), Beijing, China, August 2018.

[13] P. Zhao, X. Yang, W. Yu, J. Lin, and D. Meng, "ContextAware Multi-Criteria Handover with Fuzzy Inference in Software Defined 5G HetNets," in Proceedings of the 2018 IEEE International Conference on Communications (ICC), pp. 1-6, Kansas City, MO, USA, May 2018.
[14] O. Semenova, A. Semenov, and O. Voitsekhovska, "Neurofuzzy controller for handover operation in $5 \mathrm{G}$ heterogeneous networks," in Proceedings of the 2019 Third International Conference on Advanced Information and Communications Technologies (AICT), pp. 382-386, Lviv, Ukraine, July 2019.

[15] J. Wu, J. Liu, Z. Huang, and S. Zheng, "Dynamic fuzzy Q-learning for handover parameters optimization in $5 \mathrm{G}$ multi-tier networks," in Proceedings of the 2015 International Conference on Wireless Communications \& Signal Processing (WCSP), pp. 1-5, Nanjing, China, October 2015.

[16] A. Kose, C. H. Foh, H. Lee, and M. Dianati, "Beam-centric handover decision in dense 5G-mmWave networks," in Proceedings of the 2020 IEEE 31st Annual International Symposium on Personal, Indoor and Mobile Radio Communications, pp. 1-6, London, UK, September 2020.

[17] V. O. Nyangaresi, A. R. Joachim, and S. O. Abeka, "Neurofuzzy based handover authentication protocol for ultra dense 5G networks," in Proceedings of the 2020 Two Global Power, Energy and Communication Conference (GPECOM), pp. 339-344, Izmir, Turkey, October 2020.

[18] A. A. Khan, M. Abolhasan, W. Ni, J. Lipman, and A. Jamalipour, "A hybrid-fuzzy logic guided genetic algorithm (H-flga) approach for resource optimization in 5G VANETs," IEEE Transactions on Vehicular Technology, vol. 68, no. 7, pp. 6964-6974, 2019.

[19] A. Alhammadi, M. Roslee, M. Y. Alias, I. Shayea, S. Alriah, and A. B. Abas, "Advanced handover self-optimization approach for $4 \mathrm{G} / 5 \mathrm{G}$ HetNets using weighted fuzzy logic control," in Proceedings of the 2019 fifteenth International Conference on Telecommunications (ConTEL), pp. 1-6, Graz, Austria, July 2019.

[20] M. Gheisarnejad, M. H. Khooban, and J. Boudjadar, "Adaptive Network Based Fuzzy Inference System for Frequency Regulation in Modern Maritime Power Systems," in Proceedings of the 2019 IEEE Fifth International forum on Research and Technology for Society and Industry (RTSI), pp. 302-307, Florence, Italy, September 2019.

[21] K. Vasudeva, S. Dikmese, I. Guven, A. Mehbodniya, W. Saad, and F. Adachi, "Fuzzy-based game theoretic mobility management for energy efficient operation in HetNets," in IEEE Access, vol. 5, pp. 7542-7552, 2017.

[22] K. Vasudeva, S. Dikmese, I. Guvenc, A. Mehbodniya, W. Saad, and F. Adachi, "Fuzzy logic game-theoretic approach for energy efficient operation in HetNets," in Proceedings of the 2017 IEEE International Conference on Communications Workshops (ICC Workshops), pp. 552-557, Paris, France, May 2017.

[23] F. Bouali, K. Moessner, and M. Fitch, "A context-aware userdriven framework for network selection in 5G multi-RAT environments," in Proceedings of the 2016 IEEE Eighty fourth Vehicular Technology Conference (VTC-Fall), pp. 1-7, Montreal,Quebec,Canada, September 2016.

[24] R. R. Abdel, H. Esmaiel, and O. A. Omer, "Fuzzy logic based relay selection for MmWave communications," in Proceedings of the Ninth Annual Information Technology, Electromechanical Engineering and Microelectronics Conference (IEMECON), pp. 263-267, Jaipur, India, March 2019.

[25] K. Chatzikokolakis, P. Spapis, A. Kaloxylos, G. Beinas, and N. Alonistioti, "Spectrum sharing: a coordination framework enabled by fuzzy logic," in Proceedings of the 2015 International Conference on Computer, Information and Telecommunication Systems (CITS), pp. 1-5, Gijon, Spain, July 2015.

[26] J. G. Naragund, M. Vijayalakshmi, and S. G. Kanakaraddi, "Fuzzy Controller for Traffic Management in 5G Networks," 
in Proceedings of the 2020 IEEE fifteenth International Conference on Industrial and Information Systems (ICIIS), pp. 516-521, Rupnagar, India, November 2020.

[27] E. Skondras, A. Michalas, N. Tsolis, and D. D. Vergados, "A network selection scheme with adaptive criteria weights for 5G vehicular systems," in Proceedings of the Ninth International Conference on Information, Intelligence, Systems and Applications (IISA), pp. 1-7, Zakynthos, Greece, July 2018.

[28] S. Hongvanthong, "Novel four-layered software defined 5G architecture for AI-based load balancing and QoS provisioning," in Proceedings of the 2020 fifth International Conference on Computer and Communication Systems (ICCCS), pp. 859-863, Shanghai, China, May 2020.

[29] N. Vafamand, M. H. Asemani, T. Dragicevic, F. Blaabjerg, and M. H. Khooban, "Fuzzy-observer-based predictive stabilization of DC microgrids with power buffers through an imperfect 5G network," in IEEE Systems Journal, vol. 14, no. 3, pp. 4025-4035, 2020.

[30] M. Klymash, H. Beshley, M. Seliuchenko, and M. Beshley, "Algorithm for clusterization, aggregation and prioritization of M2M devices in heterogeneous 4G/5G network," in Proceedings of the 2017 Fourth International Scientific-Practical Conference Problems of Infocommunications. Science and Technology (PIC S\&T), pp. 182-186, Kharkov, Ukraine, October 2017.

[31] E. Skondras, E. Zoumi, A. Michalas, and D. D. Vergados, "A network selection algorithm for supporting drone services in 5G network architectures," in Proceedings of the 2019 Wireless Telecommunications Symposium (WTS), pp. 1-6, New York, USA, Apirl 2019. 